\title{
STM IMAGING OF MAGNETIC DOTS WITH FERROMAGNETIC TIP
}

\author{
A. WAWRO ${ }^{a, b}$ AND A. KASUYA ${ }^{b}$ \\ ${ }^{a}$ Institute of Physics, Polish Academy of Sciences \\ Al. Lotników 32/46, 02-668 Warsaw, Poland \\ ${ }^{b}$ Institute for Materials Research, Tohoku University \\ 2-1-1 Katahira, 980 Sendai, Japan
}

We report on imaging of isolated, few nanometers in size, magnetic dots (Co, $\mathrm{Ni}$ ) by the use of a scanning tunneling microscope equipped with a ferromagnetic Co tip in the presence of external magnetic field. The dependence of apparent height of dots as a function of magnetic field takes a hysteresis-like shape. We discuss possible mechanisms of the scanning tunneling microscope tip interactions with dots.

PACS numbers: $34.80 . \mathrm{Nz}, 36.40 . \mathrm{Cg}, 61.16 . \mathrm{Ch}$

\section{Introduction}

Contemporary trend of increasing information storage at a rate of $60 \%$ a year stimulates new research in mesoscopic magnetic systems, e.g. ferromagnetic dots. The structures which now offer writing density of order $10^{10}-10^{11} \mathrm{bits} / \mathrm{in}^{2}$ are quite easily fabricated by lithographic methods [1-3], by the use of a scanning tunneling microscope (STM) [4] and as self-organised arrays [5].

The aim of our investigations is to probe by the STM with a ferromagnetic tip the magnetization reversal in isolated magnetic particles, few nanometers in size - promising for application in high density magnetic memory. For this purpose the changes of apparent dot height in applied magnetic field are analysed.

\section{Experimental}

The whole experiment, i.e. the particle deposition and the STM observations were carried out in the vacuum $10^{-10}$ Torr at room temperature. Analyzed dots were deposited in two different ways. The first method relied on ablation from appropriate targets (Co and $\mathrm{Ni}$ ) by an excimer $\mathrm{KrF}$ laser through a quartz window. In the second one scanning of the STM tip was halted, the tip was approached towards the substrate and a pulse of voltage was applied between the STM tip and the surface. Nanoparticles were deposited onto highly oriented pyrolithic graphite (HOPG) and $\mathrm{Si}(111)$ treated chemically and thermally in a standard way. 
The STM tips were cut from $\mathrm{Co}$ and $\mathrm{Ni}$ wires and etched in $\mathrm{HCl}$. Before measurements the tips were heated in the UHV system and cleaned by scanning with high tunneling current and by applying voltage pulses. Magnetic field was induced by a coil to the maximum value of $130 \mathrm{Oe}$ in perpendicular direction to the surface. More experimental details are given in Ref. [6].

\section{Results and discussion}

Because the observed effects are expected to be a result of mutual rotation of magnetization vectors in the STM tip and analyzed dot, in the first step we carefully studied magnetoelastic properties of tips. From the measurements of tip elongation under applied magnetic field, we find that the magnetization of the $\mathrm{Ni}$ tip rotates easily and switches its direction relatively to the tip axis in $12 \mathrm{Oe}$. On the contrary, the Co tip is magnetically hard and even maximum applied field of $130 \mathrm{Oe}$ is insufficient to rotate its magnetization. It is reasonable to assume that the topmost magnetic domain on the apex of the tip, which plays most crucial role in the experiment, behaves statistically in the same way. Expecting that the magnetization of dots rotates under magnetic field, we chose for experiment magnetically hard Co tip.

Nine-point hysteresis loops presented below are a compromise between possibly detailed analysis and successful experiment. The apparent height was determined from topography images recorded in the fixed field, which was changed from a scan to scan. Other parameters as the intensity of tunneling current, the bias voltage, and feedback remained constant during a cycle of measurements. The section profiles, used for dot height determination, were parallel to the scan lines. The apparent height was measured between the highest point of the dot and the level of surrounding substrate. No interpolation procedures of studied topographic images were performed.

Figure 1 shows the dependence of apparent height of various objects on the magnetic field. The height of as deposited $\mathrm{Ni}$ dot measured without magnetic field is equal to $1.7 \mathrm{~nm}$ and its lateral size is $5 \mathrm{~nm}$. Hysteresis-like shape is very clear here (triangles). Co dot deposited from the STM Co tip on Si(111) substrate with dimensions of $10 \mathrm{~nm}$ in lateral size and $2.1 \mathrm{~nm}$ in height exhibits similar behaviour (diamonds). The apparent height also changes with the applied field. On the contrary, this effect is not observed for Co dots on Si(111) with height smaller than $1 \mathrm{~nm}$ and 3 to $5 \mathrm{~nm}$ in diameter (circles) and for Ni dots on HOPG with height equal to $0.3 \mathrm{~nm}$ [6]. The measured heights remain unchanged in the whole range of magnetic field. In order to exclude the possibility that observed dependencies of dot apparent height on magnetic field are an artifact, the height of $\mathrm{Si}(111)$ step, as an example of non-magnetic object, was measured with Co tip in the same range of magnetic field. It was measured between the fixed Si adatoms on upper and lower terraces. No traces of Si step height dependence are found (squares).

Generally, the dependence of apparent height of dot on magnetic field is found only for big particles with height larger than $1 \mathrm{~nm}$. It takes clearly hysteresis-like shape. However, the changes of the apparent height are too large to explain them only in terms of spin polarized electrons tunneling (SPET). For the 


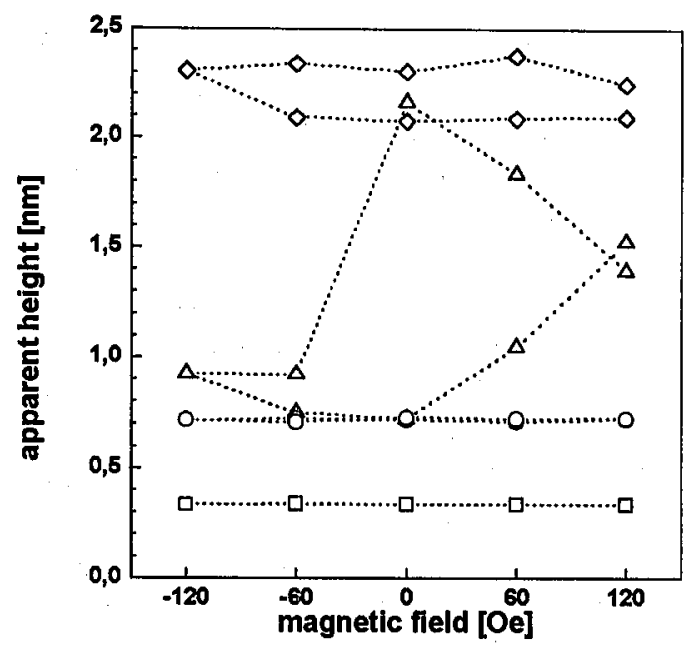

Fig. 1. The apparent height dependence on magnetic field of: $\mathrm{Ni}$ dot $1.7 \mathrm{~nm}$ in height and $5 \mathrm{~nm}$ in lateral size on HOPG (triangles), Co dot $2.1 \mathrm{~nm}$ in height and $10 \mathrm{~nm}$ in lateral size on $\mathrm{Si}$ (diamonds), Co dot $0.7 \mathrm{~nm}$ in height and $3.3 \mathrm{~nm}$ in lateral size on Si (circles), and $\mathrm{Si}$ step (squares). Dotted lines are a guide for an eye.

pure SPET phenomenon the variation of the apparent height should be of order of fraction of angström [7]. Therefore other mechanisms have to be taken into consideration. In the proximity regime dipolar interactions between magnetic moments of the tip and the sample may play an important role. The gradient of the stray fields, close to irregular objects as a dot and the apex of the tip, is higher than that for flat surfaces. The estimations of acting forces have been already performed [8] for tip-sample distances of order of a few tens of nanometer. In typical of tunneling microscope regime the forces take much higher values. In the beginning of this section we mentioned magnetoelastic effects of the STM tip observed in applied external field. During the scanning above dot both the tip and analyzed particle each other experience their strong, stray magnetic field. This field results in the change of geometrical dimensions of both magnetic objects. The orientation of magnetic vectors on the apex of the tip and a dot is determined by crystalline and shape anisotropy. However, theoretical arguments suggest that strong modifications of surface spins (their fraction in a nanoparticle is very high) may occur and substantially change the anisotropy [9]. Moreover, the atoms on the tip surface and the apex exhibit enhanced moment as compared to the bulk due to their reduced coordination number [10]. The type of substrate is another factor, which many influence properties of deposited dots.

There are strong evidences of magnetic interactions between the dots and the STM tip. The hysteresis is very clear only for dots with a height larger than $1 \mathrm{~nm}$. In the case of $\mathrm{Ni}$ dot the loop is closed. It suggests that magnetization of $\mathrm{Ni}$ dot rotates easily. The geometry of dot and the tip is unchanged during the series of scans and return to the initial tunneling conditions results in the same apparent height. The opened loop for Co dot is probably due to insufficient 
magnetic field to rotate reversibly its magnetization vector. This is consistent with magnetoelastic effects observed for magnetically hard Co STM tip. For smaller particles and for non-magnetic objects as Si substrate step there is no traces of their height changes in different magnetic field. The lack of apparent height dependence on applied magnetic field for smaller dots can be explained as a result of their superparamagnetic character.

\section{Summary}

The imaging of ferromagnetic dots few nanometers in size deposited on $\mathrm{Si}$ and HOPG substrate were studied with the use of STM equipped with ferromagnetic tips. The dependence of apparent height of dots on applied magnetic field clearly takes the shape of hysteresis. The tunneling of spin polarized electrons cannot merely explain the observed results. The magnetostatic interactions and magnetoelastic properties of the tip and dot seem to play an important role and have to be involved in the understanding of observed effects.

\section{Acknowledgments}

This work was partially supported by the Japan Society for the Promotion of Science and the Committee for Scientific Research (Poland) under project no. R- $48 / 98$.

\section{References}

[1] G. Meier, M. Kleiber, D. Grundler, D. Heitmann, R. Wiesendanger, Appl. Phys. Lett. 72, 2168 (1998).

[2] R. O'Barr, S.Y. Yamamoto, S. Schultz, W. Xu, A. Scherer, Appl. Phys. Lett. 81, $4730(1997)$.

[3] S.Y. Chou, M. Wei, P.R. Krauss, P.B. Fisher, J. Vac. Sci. Technol. B 12, 3695 (1994).

[4] A.D. Kent, S. von Molnár, S. Gider, D.D. Awschalom, J. Appl. Phys. 76, 6656 (1994).

[5] D.D. Chambliss, R.J. Wilson, S. Chiang, Phys. Rev. Lett. 66, 1721 (1991).

[6] A. Wawro, A. Kasuya, Acta Phys. Pol. A 93, 443 (1998).

[7] R. Wiesendanger, H.J. Güntherodt, G. Güntherodt, R.J. Gambino, R. Ruf, Phys. Rev. Lett. 65, 247 (1990).

[8] A. Wadas, J. Magn. Magn. Mater. 78, 263 (1989).

[9] J.C. Slonczewski, J. Magn. Magn. Mater. 117, 368 (1992).

[10] H. Ness, F. Gautier, Phys. Rev. B 52, 7352 (1995). 